\title{
Use of Buccal Oxytocin in Induction of Labour in Chinese Patients
}

\author{
H. K. CHUNG,* M.B., B.S., M.R.C.o.G.
}

Brit. med. F., 1966, 1, 710-711

The use of intravenous oxytocin infusion in "physiological doses" as advocated by Theobald et al. (1948) is one of the advances made in obstetric practice in recent years. It is a safe and effective method of induction of labour. There are, however, certain disadvantages associated with its use: (1) it immobilizes the patient; (2) some patients are frightened by the infusion apparatus; (3) the infusion may cause a local phlebitis; and (4) close and constant supervision for many hours is required. Other methods of administration of the drug have been used or suggested. Intramuscular injections, which were used extensively before intravenous infusion was introduced, have been shown to be associated with considerable risks -for example, uterine rupture. Oral administration is valueless, as oxytocin is rapidly destroyed by the gastric juice. Oxytocin is absorbed by buccal and nasal mucosa, and administration through them has been used. Dillon et al. (1960) had success with synthetic oxytocin by placing the pellets in the upper buccal sulcus on either side of the mouth, thus avoiding destruction of the drug by saliva and gastic secretion and preserving its activity. This method of administration is acceptable to the patient. It has been proved to be effective, but its safety compared with the intravenous infusion method has not yet been established. This report gives the results of an investigation into its use in Chinese patients, most of whom object strongly to intravenous infusions.

\section{Method and Administration}

It was planned to administer buccal oxytocin (Pitocin) citrate to 50 consecutive patients in Tsan Yuk Maternity Hospital who were to be induced and whose cervices were unripe. After the drug had been given to 19 patients the trial was stopped because of the risks to mother and foetus. The indications for induction in these 19 patients were as follows: toxaemia of pregnancy in 15 , post-maturity in 2 , and prolonged leakage of liquor in 2 .

The ages of the 19 patients varied from 19 to 34 (average 26), and 16 were primiparae and 3 multiparae. The maturity at the time of induction was over 37 weeks in all cases. The presentation was vertex in 18 cases and breech in one case. In all cases buccal oxytocin citrate alone was used for induction.

Oxytocin-sensitivity test (Eddie, 1963) was done in all cases to find if it could be used to predict the chances of successful induction. A $10-\mathrm{ml}$. syringe containing 0.1 international unit (I.U.) of synthetic oxytocin (Syntocinon) in $10 \mathrm{ml}$. of sterile water was used, and the needle was introduced into the antecubital vein at the ventral surface of the forearm. The solution was injected slowly and continuously at the rate of $1 \mathrm{ml}$., or 0.01 I.U. of oxytocin, in one minute with the left hand. The uterine tone was assessed by the right hand. The test was recorded as positive if a contraction occurred during the five minutes taken for the injection. A maximum dose of 0.06 I.U. was given.

Buccal administration was carried out by placing a 200-I.U. tablet of oxytocin in the parabuccal space adjacent to the upper molar teeth. The dose of oxytocin was increased 200 I.U. hourly. Therefore one additional tablet was put in the parabuccal space at the end of every hour besides replacing dissolved tablets every half-hour. The method of administration is shown in the Diagram. The induction was stopped at the end of

\footnotetext{
* Department of Obstetrics and Gynaecology, University of Hong Kong.
}

three and a half hours if there was no response, when hypertonic uterine contractions or foetal distress occurred, or when some other indication for termination of labour was present. After the onset of labour the dosage was maintained by replacing the dissolved tablets half-hourly (see Diagram). The patient was instructed not to chew or disturb the tablets, but was allowed to drink water. A medical student or a doctor stayed with the patient throughout the period of induction and labour.

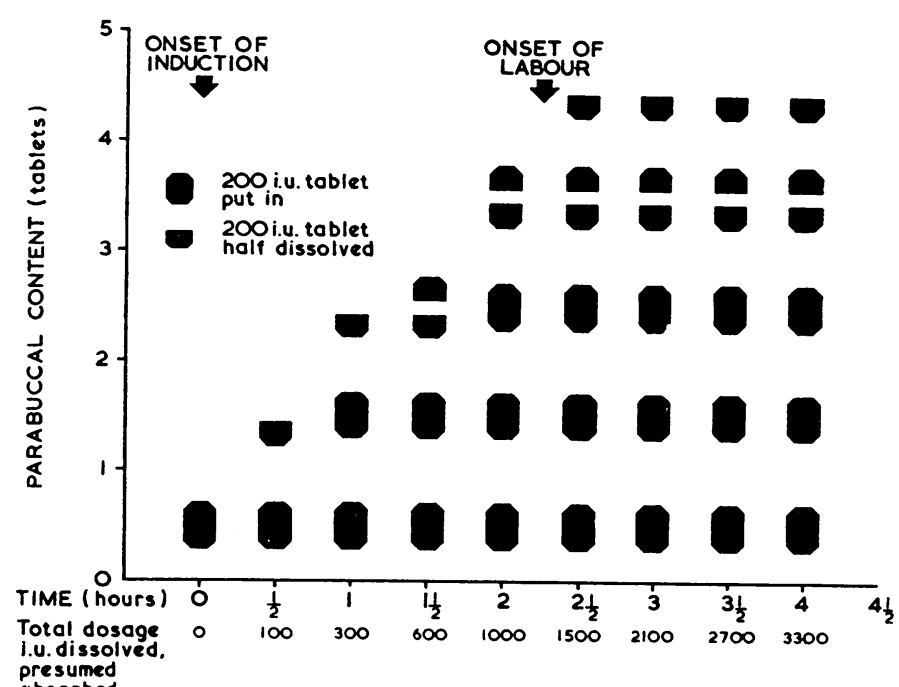

Method of administration of tablets.

\section{Results}

Labour Pains.-Regular labour pains with palpable uterine contractions started in $16(84 \%)$ of the 19 patients within 24 hours of administration of the buccal oxytocin citrate. The average induction-labour interval for the 16 patients was five hours. The average total dose of oxytocin used was 5,200 I.U. (range 2,700-11,200 I.U.). In two of the three patients in whom no response occurred the foetal heart rate dropped to less than $120 /$ minute and became irregular, though no obvious uterine contractions were felt. The induction was discontinued and caesarean section performed. The remaining patient received two courses of buccal oxytocin on consecutive days without any response and caesarean section was done. No intravenous oxytocin was given. The duration of the induction-delivery interval ranged from 3 to 48 hours (average 20.5 hours). Uterine contractions were observed to be more forceful than usual, and the first stage appeared to be shorter than that in non-induced patients. The average duration of the first stage in the patients who were delivered vaginally was 9.2 hours for primiparae and 3.8 hours for multiparae. One patient, after a total dose of 5,400 I.U. had been given, had very strong and almost continuous uterine contractions 5 hours from the beginning of induction and 2.5 hours from the onset of labour. The foetal heart rate became less than 120 and irregular. Lower-segment caesarean section was performed immediately. The baby died on the third day after birth, and post-mortem examination revealed a subdural haemorrhage.

Mode of Delivery.-Ten patients were delivered vaginally and nine by lower-segment caesarean section. The indications for caesarean section were: foetal distress in three, uterine 
dysfunction in three, tetanic uterine contractions and foetal distress in one, failed induction of labour in one, and mild cephalo-pelvic disproportion in one. The overall caesarean section rate at Tsan Yuk Hospital in 1964 was $9.8 \%$. The caesarean section rate for 84 patients in whom labour was induced by intravenous oxytocin in the three-year period 1962-4 was $30 \%$. The caesarean section rate in these 19 patients was increased $(47.4 \%)$; foetal distress or uterine dysfunction was the indication for section in most of the cases.

Maternal Mortality and Morbidity.-There was no maternal mortality, ruptured uterus, post-partum haemorrhage, or manual removal of the placenta. There was, however, an increased caesarean section rate and increased morbidity in the puerperium. The patients were happier because they were not immobilized and had no infusion apparatus beside them.

Foetal Morbidity and Mortality.-Slowing (less than 120/ minute) and irregular foetal heart rate were present in four cases during induction. Three of these babies were born in a mildly depressed state (Apgar score 3-6). One baby was in a very poor condition (Apgar score 2) when born; marked muscle-twitching and cerebral cry were present. This infant died on the third day after birth. Post-mortem examination disclosed subdural haemorrhage. The rest of the babies were born in good condition and did not require resuscitation. The perinatal mortality for this series was 52.6 per 1,000 births.

Oxytocin-sensitivity Test (Eddie, 1963). - This test was "positive" in 11 and "negative" in 8 patients. Successful induction resulted in $73 \%$ of the positive group and in $83 \%$ of the negative group. The test was also performed on 75 patients who were induced by intravenous oxytocin (Syntocinon) in physiological doses. In the 39 patients in whom the test was positive the induction success rate was $67.7 \%$, and in the 36 patients in whom the test was negative the success rate was $86 \%$. These results showed that the oxytocin-sensitivity test bore no direct relation to the result of induction by oxytocin, whether used intravenously or by the buccal method. The finding reported by Eddie (1963), that " a positive response to 0.05 unit or less of oxytocin was associated with onset of labour occurring within 48 hours of amniotomy in $91 \%$ of cases but if the test was positive to 0.03 unit or less the comparable figure was $100 \%$," was not reproduced in the-present series.

\section{Discussion}

Buccal oxytocin citrate has been shown to be effective in induction of labour (Dillon et al., 1962 ; Elstein and Wright, 1963 ; Maxwell, 1964 ; Spence and Chalmers, 1964 ; Blair, 1964). This mode of administration is acceptable to patients. Each of our 19 patients had an unripe cervix, a condition usually associated with failure of induction of labour, and yet 16 of the $19(84 \%)$ had regular labour pains after administration of buccal oxytocin citrate. The percentage of success might have been higher if the induction had not been discontinued in two patients because of foetal distress.

The question of the safety of this mode of administration is still unsettled. The dosage of the drug given is larger than that given in physiological oxytocin drip advocated by Theobald et al. (1948). The higher dose of oxytocin probably explains the occurrence of uterine hypertonicity reported by most of the observers. Dillon et al. (1962) reported one case of tetanic contractions in his series of 450 patients. Spence and Chalmers (1964) used the drug on 100 patients, and nine of them had hypertonic uterine contractions. Blair (1964) also found two cases of spasm of the uterus in the 100 patients to whom he gave the drug. In our small series of 19 cases there was one instance of hypertonic uterine contractions. Though caesarean section was performed immediately, the baby died of subdural haemorrhage. Most of our patients appeared to have a short first stage, indicating that contractions were stronger than normal. Uterine spasm or tetanic uterine contraction can occur so suddenly that even if it is detected immediately the risk to mother and foetus is still great. Leney (1964) reported one case of ruptured uterus associated with the use of buccal oxytocin, and Morgan-Jones (1964) reported two cases. Up to the present six cases of ruptured uterus have been reported in association with the use of buccal oxytocin. Therefore it has definite risks. The dosage is large. It is probable that "linguets" containing less than 200 I.U. might give equally good results with fewer risks.

In our series there was a high operative delivery rate, attributed partly to the complications that had called for induction and partly to an increased proportion of foetal distress and dysfunctional uterine contractions, which were known to be associated with this method of induction. Further investigations and possibly modifications of the dosage of buccal oxytocin citrate "linguets" might give useful information. The cases must be chosen with care, and constant medical supervision of the patients during induction is required. All induction must be done in a hospital equipped for caesarean section. The hope that buccal oxytocin citrate is a safe drug for the patient to take at home has not yet been fulfilled.

In view of the high operative delivery rate and the occurrence of tetanic uterine contraction, the trial was stopped before a statistically comparable series of cases of induction of labour by intravenous and by buccal oxytocin was collected. We have used intravenous infusion of oxytocin in physiological doses in 84 patients in the past three years. No case of tetanic uterine contraction or ruptured uterus was encountered. The caesarean section rate was $30 \%$. There were three stillbirths among these 84 patients and no neonatal deaths. In two of these stillbirths the foetus was dead before induction, and the remaining one was a case of anencephaly. The impression is that intravenous oxytocin in physiological doses is a safer procedure than buccal oxytocin.

\section{Summary}

Labour was successfully induced in $16(84 \%)$ of the 19 patients who were given buccal oxytocin (Pitocin) citrate. The average total dose of buccal oxytocin required was 5,200 I.U. There was one case of hypertonic uterine contractions, and the baby died of subdural haemorrhage. Nine (47\%) were delivered by lower-segment caesarean section. Foetal distress was detected in four patients during induction. The perinatal mortality for this series was 52.6 per 1,000 births. An oxytocinsensitivity test (Eddie, 1963) was performed in all patients in this series. It was found that one could not rely on the test for prediction of the success of induction. The complications associated with this method of induction are discussed. Buccal oxytocin is a potentially valuable method of induction of labour, but the dosage and the rate of absorption of the tablet in its present form need further modification.

I wish to express my thanks to Professor Daphne W. C. Chun and Miss G. H. Dodds for their advice, criticism, and encouragement; to the medical and nursing staff, especially Dr. K. H. Ng, in Tsan Yuk Hospital, who gave me their co-operation and help; and to Parke Davis and Company, who supplied the buccal Pitocin citrate "linguets."

\section{REFERENCES}

Blair, R. G. (1964). Lancet, 1, 637.

Dillon, T. F., Douglas, R. G., Du Vigneaud, V., and Barber, M. L. (1960). Obstet. and Gynec., 15, 587.

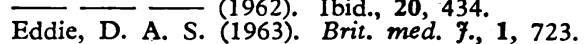

Edstein, M., and Payling Wright, H. (1963). 7. Obstet. Gynaec. Brit. Cwlth, 70, 1005.

Leney, P. M. (1964). Brit. med. 7., 2, 689.

Maxwell, A. W. (1964). F. Obstet. Gynaec. Brit. Cwlth, $71,37$.

Morgan-Jones, D. V. (1964). Brit. med. F., 2, 874.

Spence, D. N., and Chalmers, J. A. (1964). Lancet, 1, 633.

Stewart, D. B., and Bernard, R. M. (1954). F. Obstet. Gynaec. Brit. Emp., 61, 318 .

Theobald, G. W., Graham, A., Campbell, J., Gange, P. D., and Driscoll W. J. (1948). Brit. med. Ұ., 2, 123. 